\title{
Fukushima Daiichi Information Repository FY13 Status
}

Curtis Smith Cherie Phelan Dave Schwieder

September 2013

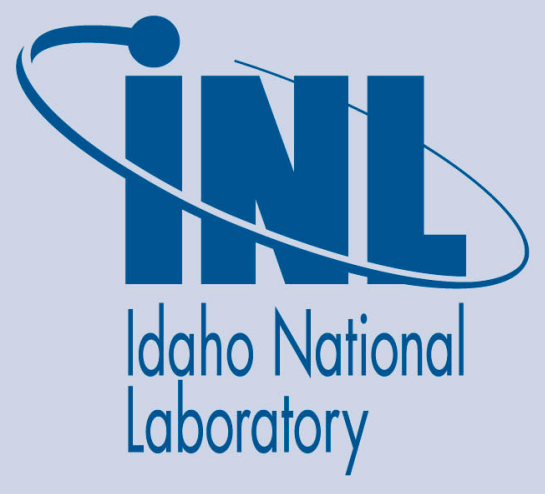

The INL is a U.S. Department of Energy National Laboratory operated by Battelle Energy Alliance 
INL/EXT-13-30234

\title{
Fukushima Daiichi Information Repository FY13 Status
}

\author{
Curtis Smith \\ Cherie Phelan \\ Dave Schwieder
}

September 2013

\section{Idaho National Laboratory \\ Idaho Falls, Idaho 83415}

http://www.inl.gov

\author{
Prepared for the \\ U.S. Department of Energy \\ Office of Nuclear Energy \\ Under DOE Idaho Operations Office \\ Contract DE-AC07-05ID14517
}




\section{Introduction}

The accident at the Fukushima Daiichi nuclear power station in Japan is one of the most serious in commercial nuclear power plant operating history. Much will be learned that may be applicable to the U.S. reactor fleet, nuclear fuel cycle facilities, and supporting systems, and the international reactor fleet. For example, lessons from Fukushima Daiichi may be applied to emergency response planning, reactor operator training, accident scenario modeling, human factors engineering, radiation protection, and accident mitigation; as well as influence U.S. policies towards the nuclear fuel cycle including power generation, and spent fuel storage, reprocessing, and disposal.

This document describes the database used to establish a centralized information repository to store and manage the Fukushima data that has been gathered. The data is stored in a secured (password protected and encrypted) repository that is searchable and available to researchers at diverse locations.

In FY13, the Idaho National Laboratory extended the Information Portal (found at https://fukushima.inl.gov) to allow for a "public access" view that contains non-proprietary information.

\section{Overview of the Information Portal}

Central to the data base design is the concept of an Event. It is the sequence of these events that will characterize the accident as it unfolded in Japan. The following dependency statements define the type of information being collected and how the data is related. These statements are used as a starting point for deriving data base schema. 
An example of an event is shown in Figure 1.

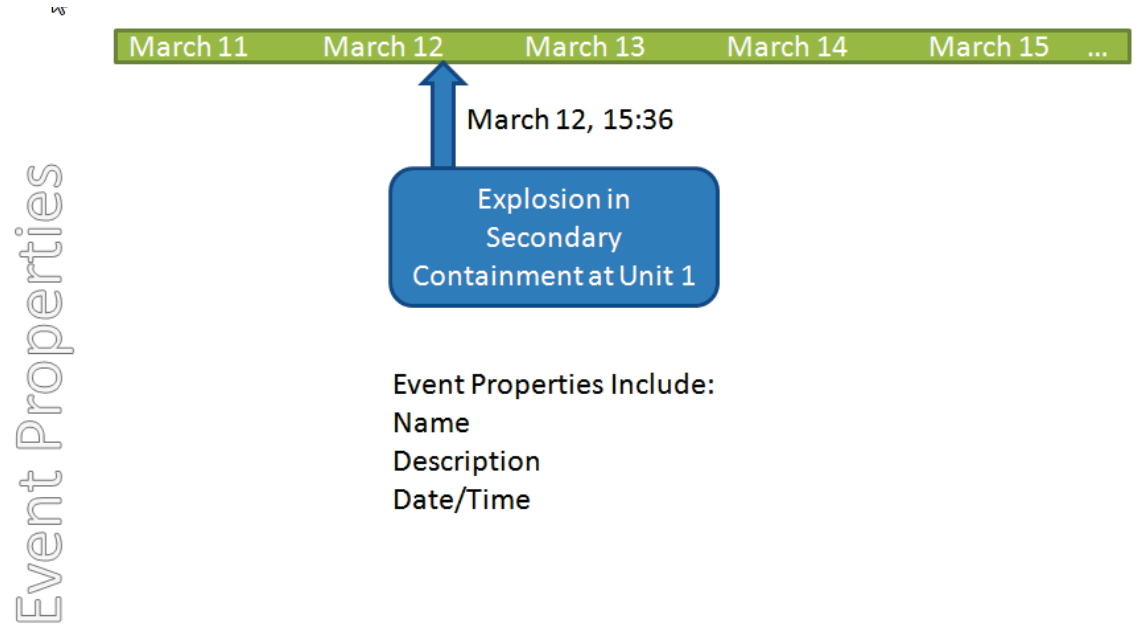

Figure 1. Example of an event.

1. An EVENT can have zero to many "Artifacts" that support the event's attributes.

An example of an EVENT associated with plant parameter(s) is shown in Figure 2.

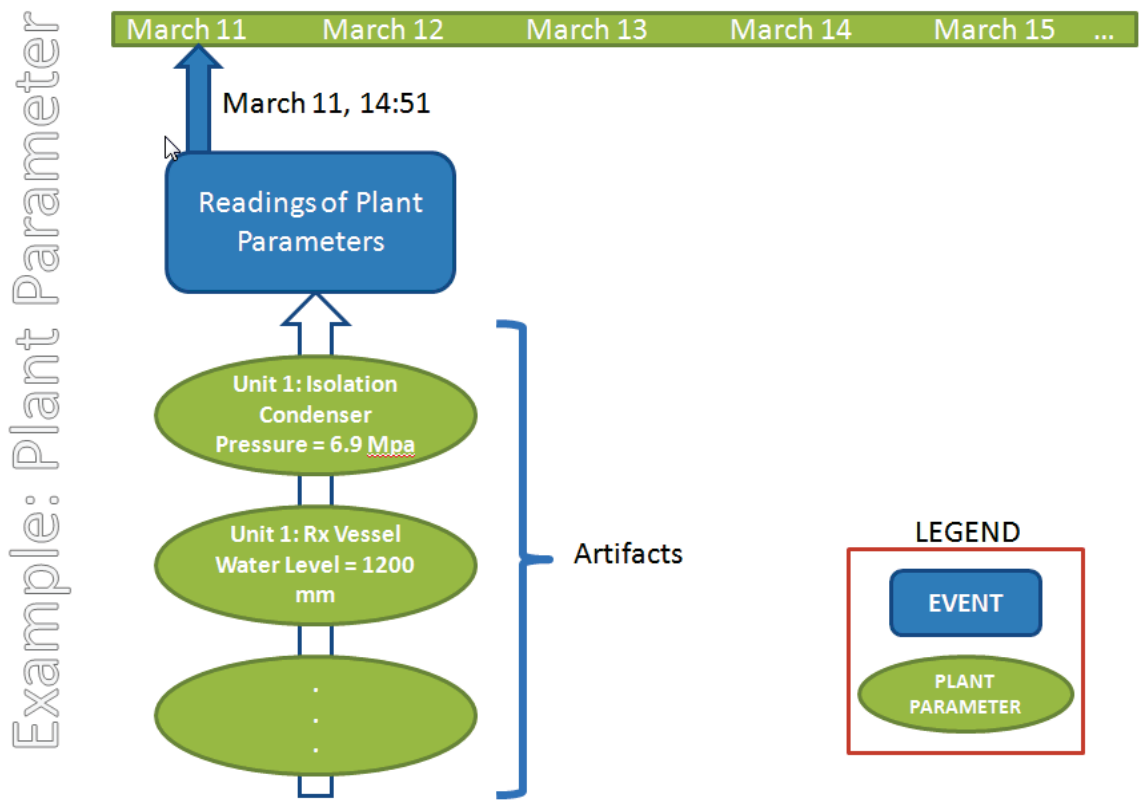

Figure 2. Event associated with plant parameters. 
An example of an EVENT associated with media file(s) is shown in Figure 3.

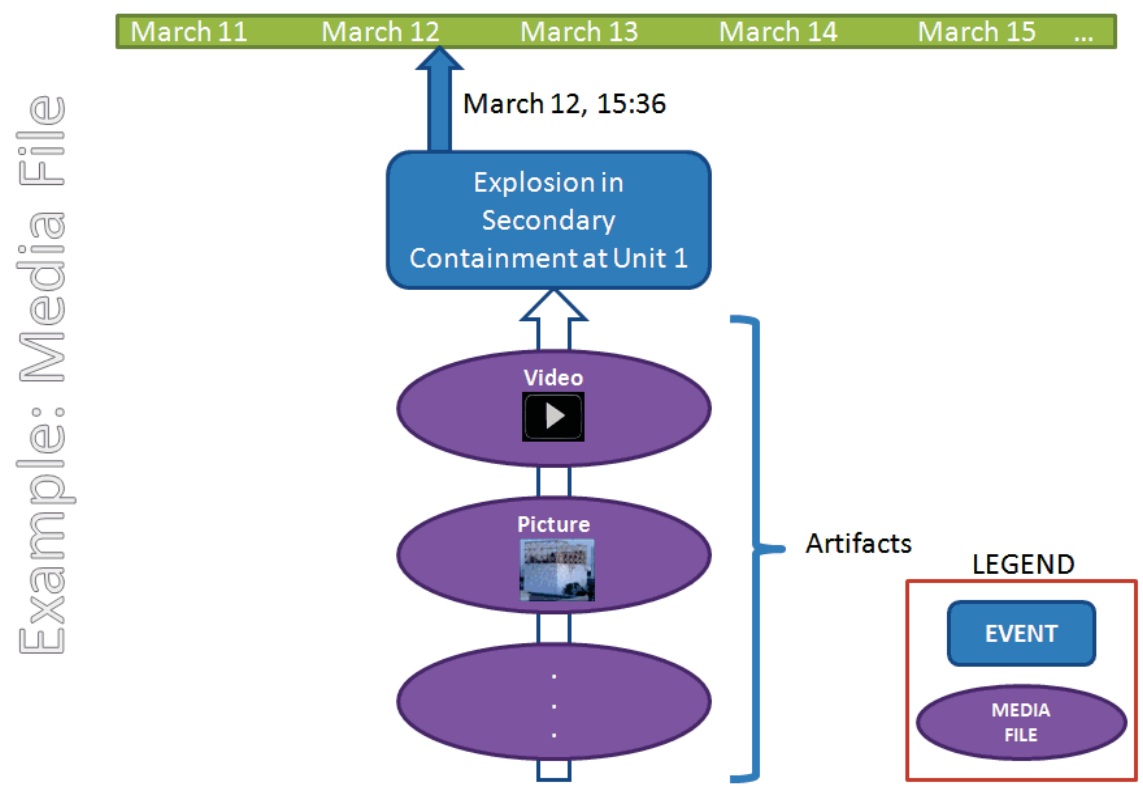

Figure 3. Event associated with media files.

An example of an EVENT associated with component state(s) is shown in Figure 4.

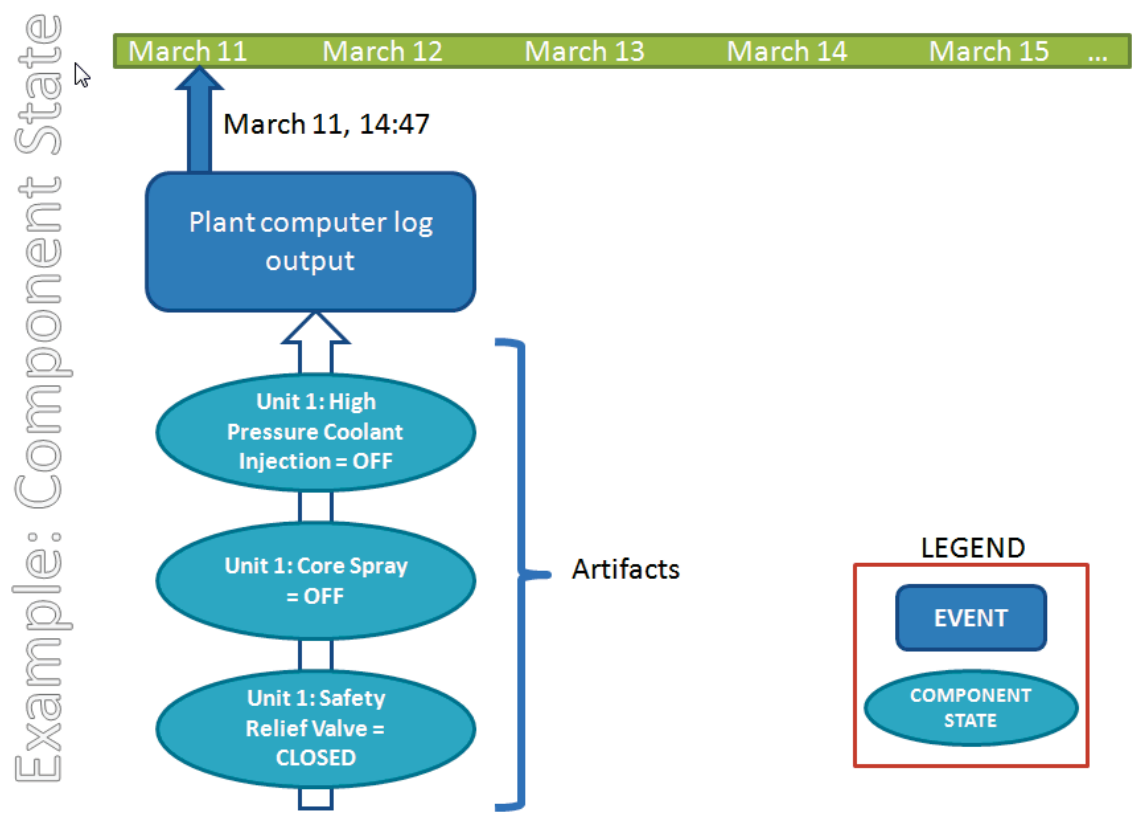

Figure 4. Event associated with plant parameters. 
An example of an EVENT associated with multiple artifact types is shown in Figure 5.

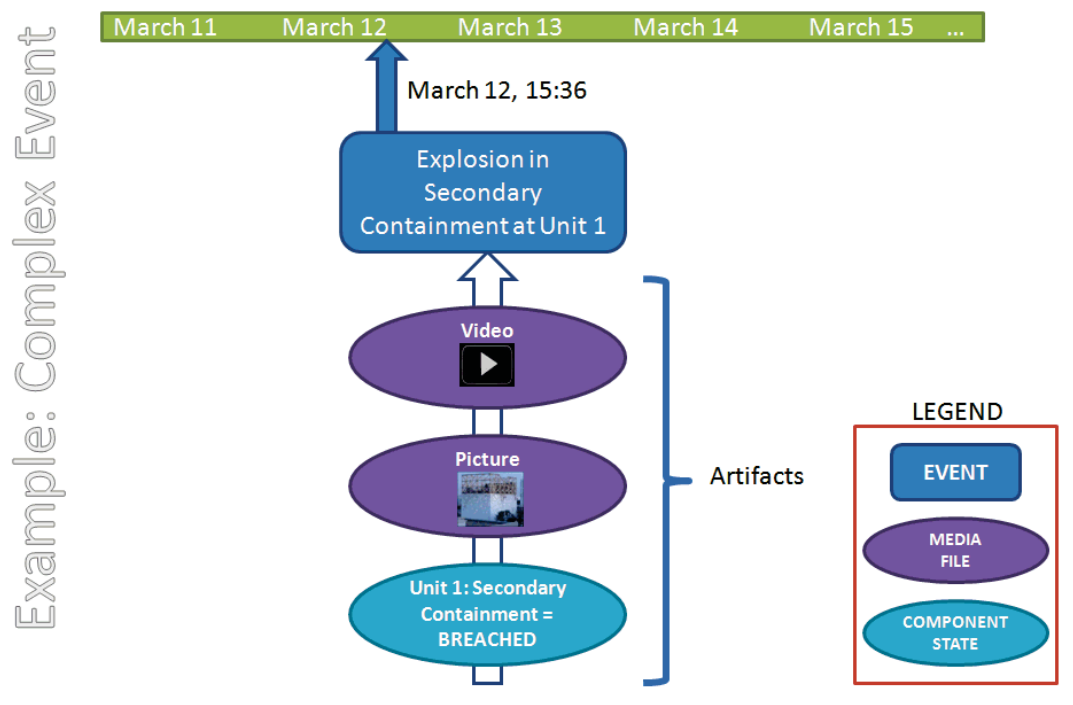

Figure 5. Event associated with multiple artifacts.

\section{Roles used by the Information}

The following roles will available: Administrator, Contributor, Pending, Public, Reviewer and User.

Administrator: This role has access to only the Home screen, Help, and Admin menu items which includes the Manage User Accounts screen.

Contributor: This role has access to the Home, Events, Artifacts, and Help screens. The Reviews will be visible to this role from the Events and Artifacts screens but they ability to Add, Modify and Delete Reviews will not be available, but the ability to Add, Modify, and Delete Events/Artifacts will be available.

Pending: The Pending role is assigned when a user initially registers. This role remains assigned until an administrator. The user cannot log in until an administrator updates his roles.

Public: This role has access to the same screens as the User role, but with further limitations on to what data is displayed. 
Reviewer: This role has access to the Home screen, Help, Events, Artifacts, and the Review menu items which includes the Review Artifacts and Review Events screens. However, the Events and Artifacts screens are accessible but without the Add, Modify, and Delete Event/Artifact functionality, but with the Add, Modify, Delete Review functionality.

User: This role has access to the Home screen, Help screen, and the Events and Artifacts screen. However, the Events and Artifacts screens are accessible only for viewing - no updates can be made.

The table below shows the roles and what access the each role will have.

\begin{tabular}{|l|c|c|c|c|c|c|c|c|}
\hline Role & Home & Events & Artifacts & Reviews & Views & Tools & Admin & Help \\
\hline & & & & & & & & \\
\hline Administrator & $\mathrm{X}$ & & & & & & $\mathrm{X}$ & $\mathrm{X}$ \\
\hline Contributor & $\mathrm{X}$ & $\mathrm{X}$ & $\mathrm{X}$ & & & & & $\mathrm{X}$ \\
\hline Pending & & & & & & & & $\mathrm{X}$ \\
\hline Public & $\mathrm{X}$ & $\mathrm{X}$ & $\mathrm{X}$ & & $\mathrm{X}$ & & & $\mathrm{X}$ \\
\hline Reviewer & $\mathrm{X}$ & $\mathrm{X}$ & $\mathrm{X}$ & $\mathrm{X}$ & & & & $\mathrm{X}$ \\
\hline User & $\mathrm{X}$ & $\mathrm{X}$ & $\mathrm{X}$ & & $\mathrm{X}$ & & & $\mathrm{X}$ \\
\hline
\end{tabular}

\section{FY13 Activities}

A variety of maintenance activities were conducted in FY13 on the Information Portal, including changes to the website:

- Addition of a contact page that allows a message as well as files to be sent to the INL

- A "most recent flag" that can be added to the artifact so they can be filtered to show only the most recent TEPCO information

- Public access to the website (approximately 300 public accesses have been made since this option was enabled)

- Updated information such as the report on Ex-vessel Analysis for Unit 1.

\section{Current Status}

The following figures are examples of how the Information Portal currently looks. Just a subset of the portal screens is shown. 
The initial screen welcomes the user:

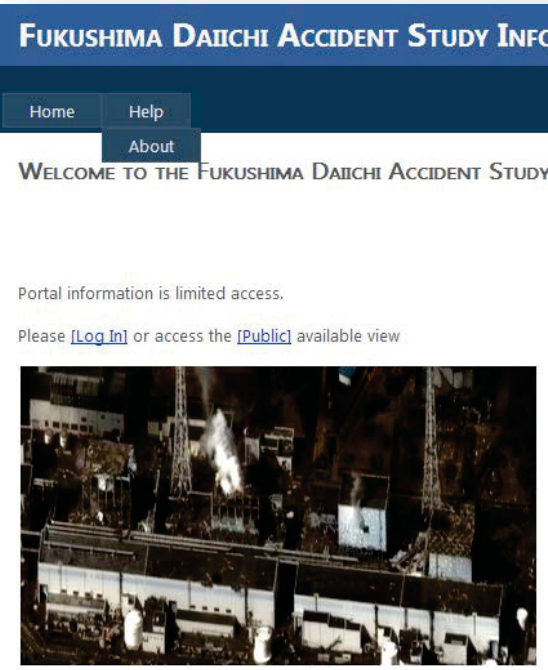

Entering the public view will display:

Fukushima Daitchi Accident Study Information Portal (Public View)

\section{\begin{tabular}{l|l|l|l|l}
\hline Home & Events & Artifacts & Views & Contact Us \\
\hline
\end{tabular}}

Welcome to the Fukushima Dailchi Accident Study Information Portal

The study is available at this link: Eukushima Daiichi Accident Study

The latest portal report is available at this link: Fukushima Daiichi Accident Study Information Portal Report

Further evaluations of core debris modeling at this link: Ex-vessel Analysis for Unit 1

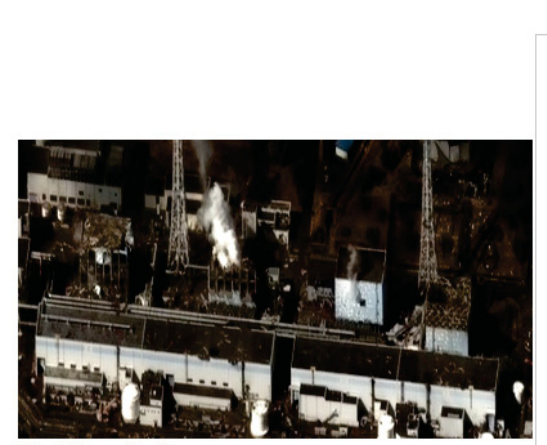

Recent Additions/Changes

View Information for..

Unit 1 Timeline

Unit 2 Timeline

Unit 3 Timeline

Unit 4 Timeline

Unit 6 Timeline

\begin{tabular}{|c|c|c|c|}
\hline & Date/Time & Type & Description \\
\hline Details & $\begin{array}{l}\text { 3/25/2013 } \\
\text { 3:34:52 PM }\end{array}$ & Artifact & $\begin{array}{l}\text { Unit } 1 \text { Reactor Building } \\
\text { radiation survey... }\end{array}$ \\
\hline Details & $\begin{array}{l}\text { 2/1/2013 } \\
\text { 3:21:48 PM }\end{array}$ & Artifact & $\begin{array}{l}\text { Transient recorder data unit } \\
3\end{array}$ \\
\hline Details & $\begin{array}{l}\text { 2/1/2013 } \\
3: 20: 11 \text { PM }\end{array}$ & Artifact & $\begin{array}{l}\text { Chart recorder parameter } \\
\text { data unit } 3\end{array}$ \\
\hline Details & $\begin{array}{l}\text { 2/1/2013 } \\
\text { 3:19:27 PM }\end{array}$ & Artifact & $\begin{array}{l}\text { Updated temperature data } \\
\text { unit } 3\end{array}$ \\
\hline Details & $\begin{array}{l}\text { 2/1/2013 } \\
\text { 3:18:56 PM }\end{array}$ & Artifact & $\begin{array}{l}\text { Updated water level and } \\
\text { pressure data un... }\end{array}$ \\
\hline Details & $\begin{array}{l}\text { 2/1/2013 } \\
3: 18: 02 \text { PM }\end{array}$ & Artifact & $\begin{array}{l}\text { Updated component state } \\
\text { data unit } 3\end{array}$ \\
\hline Details & $\begin{array}{l}\text { 2/1/2013 } \\
\text { 3:17:33 PM }\end{array}$ & Artifact & $\begin{array}{l}\text { Updated event timeline data } \\
\text { unit } 3\end{array}$ \\
\hline Details & $\begin{array}{l}\text { 2/1/2013 } \\
\text { 3:15:34 PM }\end{array}$ & Artifact & $\begin{array}{l}\text { Transient recorder data unit } \\
2\end{array}$ \\
\hline Details & $\begin{array}{l}\text { 2/1/2013 } \\
\text { 3:14:35 PM }\end{array}$ & Artifact & $\begin{array}{l}\text { Parameter chart recorder } \\
\text { data unit } 2\end{array}$ \\
\hline
\end{tabular}


Entering the Events portion of the Portal will provide access to detailed events during the Fukushima accident:

\begin{tabular}{|c|c|c|c|c|c|c|c|}
\hline \multicolumn{7}{|c|}{ Fukushima Daitchi Accident Study Information Portal (Public View) } & \multirow[t]{2}{*}{$\underline{\log I n]}$} \\
\hline Events & Artifacts & Views & Contact & Help & & & \\
\hline \multicolumn{7}{|l|}{ Additional Filters: } & \\
\hline Area Unit1 & $\nabla$ & Artifact Type & Media File & $\nabla$ Confidence & e At Least All & & \\
\hline \multicolumn{8}{|l|}{ Events } \\
\hline $\begin{array}{l}\text { Start } \\
\text { Date/Time }\end{array}$ & $\begin{array}{l}\text { End } \\
\text { Date/Time }\end{array}$ & \multirow{2}{*}{\multicolumn{2}{|c|}{ Event }} & \multirow[t]{2}{*}{ Description } & Cause & $\begin{array}{l}\text { Change } \\
\text { Date/Time }\end{array}$ & \\
\hline All $\quad \longrightarrow$ & All $\quad \longrightarrow$ & & & & All - & $\nabla$ All $\square$ & \\
\hline \multicolumn{7}{|c|}{$1 \underline{2}$} & \\
\hline Sort & Sort & Sort & & Sort & Sort & Sort & \\
\hline$\nabla_{2: 46: 00 \mathrm{PM}}^{3 / 11 / 2011}$ & $\begin{array}{l}\text { 3/11/2011 } \\
\text { 2:46:00 PM }\end{array}$ & \multicolumn{2}{|c|}{ Earthquake (Tohoku) } & $\begin{array}{l}\text { A major earthquake occurred } \\
\text { located off the coast of the } \\
\text { Miyagi Prefecture. }\end{array}$ & Earthquake & $\begin{array}{l}5 / 24 / 2012 \\
10: 02: 55 \text { AM }\end{array}$ & \\
\hline $\begin{array}{l}\text { 3/11/2011 } \\
2: 46: 00 \mathrm{PM}\end{array}$ & $\begin{array}{l}\text { 3/11/2011 } \\
\text { 2:46:00 PM }\end{array}$ & \multicolumn{2}{|c|}{ Unit 1 Shut Down } & $\begin{array}{l}\text { Reactor Scram, Turbine Trip, all } \\
\text { control rods full... }\end{array}$ & Automated Action & $\begin{array}{l}\text { 8/22/2011 } \\
4: 26: 00 \mathrm{PM}\end{array}$ & \\
\hline$\square \begin{array}{l}3 / 11 / 2011 \\
2: 47: 00 \text { PM }\end{array}$ & $\begin{array}{l}\text { 3/11/2011 } \\
\text { 2:47:00 PM }\end{array}$ & \multicolumn{2}{|c|}{ Loss of Offsite Power } & Loss of all offsite power sources. & Loss of Power & $\begin{array}{l}\text { 8/23/2011 } \\
\text { 12:18:51 PM }\end{array}$ & \\
\hline$\square$ 3:102:00 PM & $\begin{array}{l}\text { 3/11/2011 } \\
\text { 3:02:00 PM }\end{array}$ & \multicolumn{2}{|c|}{$\begin{array}{l}\text { Unit } 1 \text { subcriticality } \\
\text { confirmed }\end{array}$} & Reactor subcriticality confirmed. & Operator Action & $\begin{array}{l}\text { 10/25/2011 } \\
\text { 9:50:59 AM }\end{array}$ & \\
\hline$\square$ 3/11/2011 & $\begin{array}{l}\text { 3/11/2011 } \\
\text { 3:27:00 PM }\end{array}$ & \multicolumn{2}{|c|}{$\begin{array}{l}\text { 1st Tsunami wave hits } \\
\text { unit } 1\end{array}$} & $\begin{array}{l}\text { Tsunami Wave generated by the } \\
\text { earthquake strikes t... }\end{array}$ & Earthquake & $\begin{array}{l}\text { 10/25/2011 } \\
8: 38: 49 \text { AM }\end{array}$ & \\
\hline$\square$ 3:11/2011 & $\begin{array}{l}\text { 3/11/2011 } \\
\text { 3:35:00 PM }\end{array}$ & \multicolumn{2}{|c|}{$\begin{array}{l}\text { 2nd Tsunami wave hits } \\
\text { unit } 1\end{array}$} & $\begin{array}{l}\text { Tsunami Wave generated by the } \\
\text { earthquake strikes t... }\end{array}$ & Earthquake & $\begin{array}{l}\text { 10/25/2011 } \\
\text { 8:40:02 AM }\end{array}$ & \\
\hline $\begin{array}{l}\text { 3/11/2011 } \\
3: 37: 00 \text { PM }\end{array}$ & $\begin{array}{l}\text { 3/11/2011 } \\
\text { 3:37:00 PM }\end{array}$ & \multicolumn{2}{|c|}{ Station Blackout, Unit 1} & $\begin{array}{l}\text { Loss of off site and on site } A C \\
\text { power sources }\end{array}$ & Tsunami Flooding & $\begin{array}{l}\text { 10/25/2011 } \\
\text { 8:43:42 AM }\end{array}$ & \\
\hline$\square=3 / 11 / 2011$ & 3/11/2011 & \multicolumn{2}{|c|}{ MCR lit by temporary } & Main Control Room lit by & Operator Action & 11/2/2011 & \\
\hline
\end{tabular}

Entering the Artifacts portion of the Portal will provide access to stored files such as reports and pictures:

\begin{tabular}{|c|c|c|c|}
\hline \multicolumn{3}{|c|}{ Fukushima Daitchi Accident Study Information Portal (Pubuc View) } & \multirow[t]{2}{*}[\operatorname{log}\text{In}]{} \\
\hline Events & contact Us & Help & \\
\hline \multicolumn{3}{|r|}{ 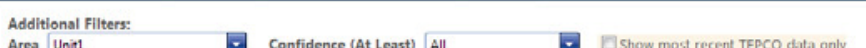 } & \\
\hline \multicolumn{4}{|l|}{ Artifacts } \\
\hline Description & Source $\quad 9$ & $\begin{array}{l}\text { Type } \\
\begin{array}{l}\text { Start Date/Time } \\
\text { Media file }\end{array}\end{array}$ & \\
\hline Sont & Sort & Sort sort & \\
\hline $\begin{array}{l}\text { Report of the Japanese } \\
\text { Government to the LAEA } \\
\text { Ministerial Conference on } \\
\text { Nuclear Safety. Chapter iv. }\end{array}$ & $\begin{array}{l}\text { Nuclear Emergency } \\
\text { Response } \\
\text { Headquarters, } \\
\text { Government of } \\
\text { Japan, (June 2011) } \\
\end{array}$ & Media Flle & \\
\hline$\square$ Damage to unit 1 after hydrogen & ${ }^{n}$ ISIS & Media file & \\
\hline $\begin{array}{l}\square \text { Additional Report of the } \\
\text { Japanese Government to th.. }\end{array}$ & $\begin{array}{l}\text { Second report from } \\
\text { the Government of } \\
\text { Japan to the - }\end{array}$ & Media file & \\
\hline$\square$ Chronology of Main Events at & Tepco Report & Media Flle & \\
\hline $\begin{array}{l}\square \text { Fulushima Daiichi Nuclear Power } \\
\text { Station Unit } 1 \mathrm{Ci} \text {... }\end{array}$ & TEPCO Report & Media file & \\
\hline
\end{tabular}




\section{Entering the Views portion of the Portal will provide several different ways to look at information stored in the Portal.}

\section{Timeline}

\begin{tabular}{|c|c|c|c|c|}
\hline 3/11/2011 12:00 PM & 㖆 () & 3/18/2011 12:00 PM & 嘈 Q & First event +7 days \\
\hline \multirow[t]{3}{*}{ Generate Timeline } & \multirow{3}{*}{\multicolumn{3}{|c|}{ Generate for Printer }} & Earthquake to last event \\
\hline & & & & Complete timeline span \\
\hline & & & & Set Manually \\
\hline
\end{tabular}

Timeline for Unit1

$\Xi$ Friday, March 11, 2011

12.00.00 PM - Isolation Condenser - Actuation Train - IC A - Standby

12.00.00 PM - High Pressure Coolant Injection - Turbine driven pump - HPCI - Standby

12.00.00 PM - Service Water System - Motor driven pump - CCSW - Operating

12.00.00 PM - DC Power - Battery - $125 \mathrm{~V}$ Battery - Operating

12.00.00 PM - Power Conversion - Main steam isolation valve - MSIVs - Normally Open; Not failed

12.00.00 PM - Offsite Electrical Power - System level event - Offsite Power Sources - Operating

12.00.00 PM - Reactor Protection - Actuation Train - Reactor - Operating

12.00.00 PM - AC Power System - Emergency diesel generator - EDG 2 - Standby

12.00.00 PM - AC Power System - Emergency diesel generator - EDG 1 - Standby

$\boxplus$ 2.46.00 PM - Earthquake (Tohoku)

$\boxplus$ 2.46.00 PM - Unit 1 Shut Down

$\boxplus$ 2.46.01 PM - Reactor Protection - Actuation Train - Reactor - Reactor Scram

Ð 2.47.00 PM - Offsite Electrical Power - System level event - Offsite Power Sources - No power-loss of power

$\boxplus$ 2.47.00 PM - Power Conversion - Main steam isolation valve - MSIVs - Normally open; fail in closed position

$\boxplus$ 2.47.00 PM - AC Power System - Emergency diesel generator - EDG 2 - Automatically Started

$\boxplus$ 2.47.00 PM - AC Power System - Emergency diesel generator - EDG 1 - Automatically Started

$\boxplus$ 2.47.00 PM - Loss of Offsite Power

2.52.00 PM - Isolation Condenser - Actuation Train - IC B - Automatically Started

$\boxplus$ 2.52.00 PM - Isolation Condenser - Actuation Train - IC A - Automatically Started

Ð 3.02.00 PM - Unit 1 subcriticality confirmed

3.03.00 PM - Isolation Condenser - Actuation Train - IC B - Manually Shutdown

$\boxplus$ 3.03.00 PM - Isolation Condenser - Actuation Train - IC A - Manually Shutdown

$\boxplus$ 3.27.00 PM - 1st Tsunami wave hits unit 1 


\section{Graphical Timeline}

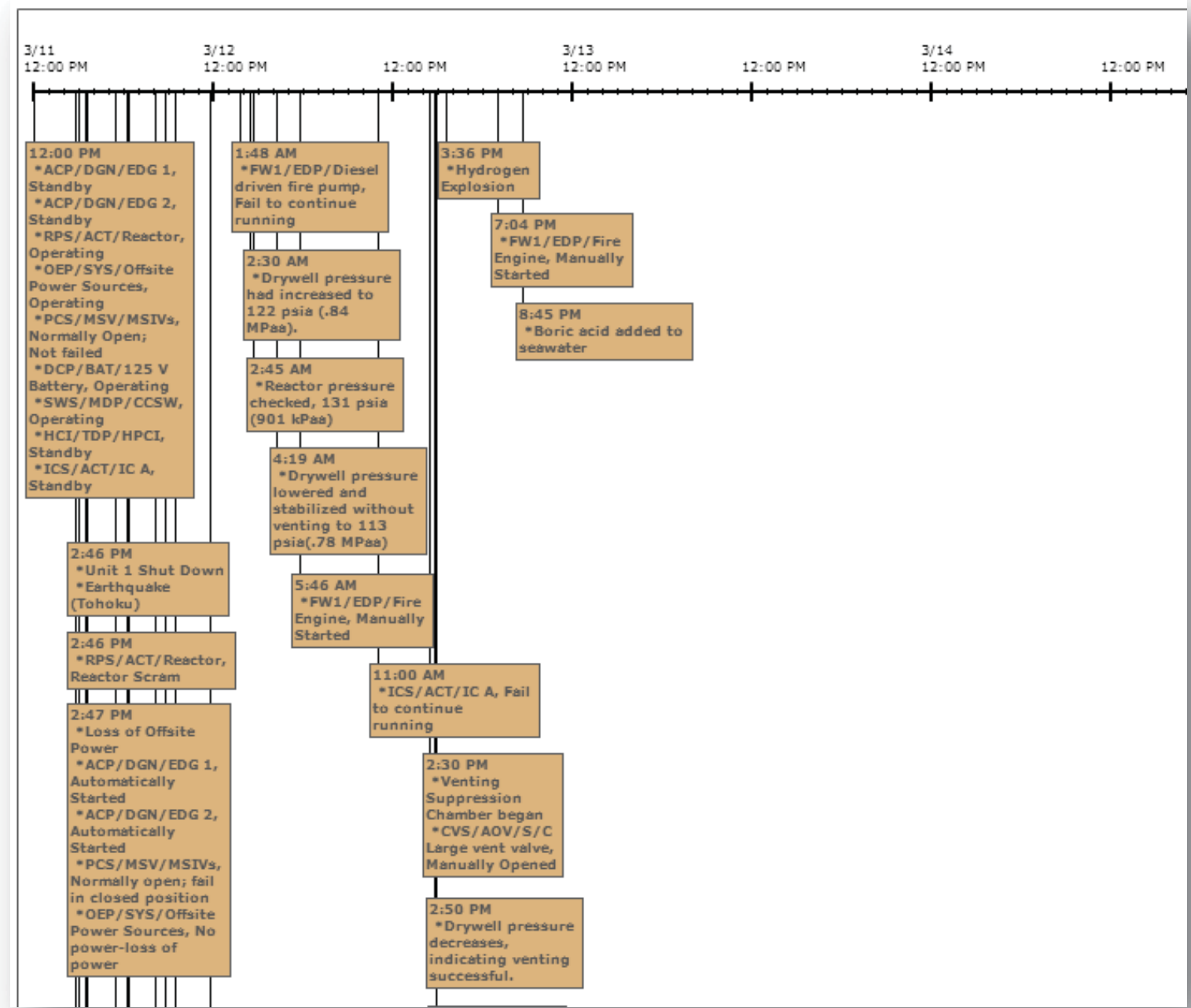

